\title{
Scenario Based Transmission Power Control (TPC) Analysis over Wireless Body Area Network (WBAN)
}

\author{
Taslim Arefin ${ }^{1 \#^{*},}$ Mohammad Hanif Ali ${ }^{1}$, A. K. M. Fazlul Haque ${ }^{2}$ \\ ${ }^{1}$ Department of CSE, Jahangirnagar University, Dhaka, Bangladesh \\ ${ }^{2}$ Department of ETE, Daffodil International University, Dhaka, Bangladesh \\ Email: *arefin@diu.edu.bd
}

How to cite this paper: Arefin, T., Ali, M.H. and Haque A.K.M.F. (2021) Scenario Based Transmission Power Control (TPC) Analysis over Wireless Body Area Network (WBAN). Communications and Network, 13, 93-110.

https://doi.org/10.4236/cn.2021.133008

Received: March 20, 2021

Accepted: August 23, 2021

Published: August 26, 2021

Copyright $\odot 2021$ by author(s) and Scientific Research Publishing Inc. This work is licensed under the Creative Commons Attribution International License (CC BY 4.0).

http://creativecommons.org/licenses/by/4.0/

\begin{abstract}
In the modern age, Wireless Body Area Network (WBAN) becomes very popular everywhere for monitoring healthcare services remotely. However, the WBAN system has lagged in efficient power consumption till now. As WBAN is formed with several portable devices, low power consumption will ensure battery lifetime. In this paper, an analysis of Transmission Power Control (TPC) over WBAN has been conducted. A ZigBee based WBAN model with different network topologies and data rates has been proposed in the experiment. WBAN data-management technique has been proposed due to reducing more data transmission. Less data transmission reduces overall power consumption. The whole work has been done using OPNET and OMNET++ network simulators. Six sensor nodes have been used with a ZigBee coordinator in the simulation scenario where throughput, load, delay, data traffic, amount of power consumption, packet delivery ratio, etc. have been used as simulation parameters. TPC analysis indicates the power consumptions in different topologies, with different data rates. Several simulation scenarios were run and the results were analyzed in this paper.
\end{abstract}

\section{Keywords}

WBAN, ZigBee, TPC, Power Control, Transmission Power

\section{Introduction}

Through modern-day technology, services and applications, it has become easy to save human lives via providing far better monitoring for health care services. Thus, 
electronic health care was created to serve such a purpose, the system has several health care applications and it's referred to as the e-health system [1]. Furthermore, this technology can provide a better health care service for the patient's everyday status, by creating associate increased manner of observation and taking care of patients in their natural setting at their homes or anyplace else in each day's life. Also, the exploitation the wireless technology can give associate improved period observation within the future [2]. Being aforementioned that, wireless body area network (WBAN) consists of a variety of sensors that area unit either ingrained within the patient's body or wearable convenience as an example a monitor watch, to live that person's health stats. Additionally, it's vital to say here that sensors should be made by considering the subsequent features: lightweight Wight, small size, optimum power consumption, and low value [3]. Figure 1 shows a wireless body area network. Moreover, the sensors area unit referred to as nodes within the system and the area unit connected to a getaway, the entranceway is accountable being an instrumentality to the network, helping to transfer information to the medical server and store it within the patient's file [4]. It's vital to say that, WBAN is taken into account one of the still rising technologies beneath its progress phases associated it's beneath an early stage of development, and it is expected to be at some point a stable system utilized in the health care applications.

WBAN has recently received a lot of attention for its application to the assisted remote patient observance and living. WBAN contains wireless devices that are secured to the soma to observe a large variety of physical quantities. The character of those wireless sensors permits nice flexibility in their movement. New sector square measure gap for these technologies in medical and health observance service, e.g. period mensuration of pulse, body temperature, and pressure level, etc. Analysis of life signals in patients with chronic conditions [5].

Like Wireless Sensor Networks (WSNs) applications, the power consumption of WBAN nodes is so essential. In health observance, all sensor nodes should operate for a long period of time as a result of charging isn't potential and also the choice of optimum transmission power becomes vital. Several WBAN applications

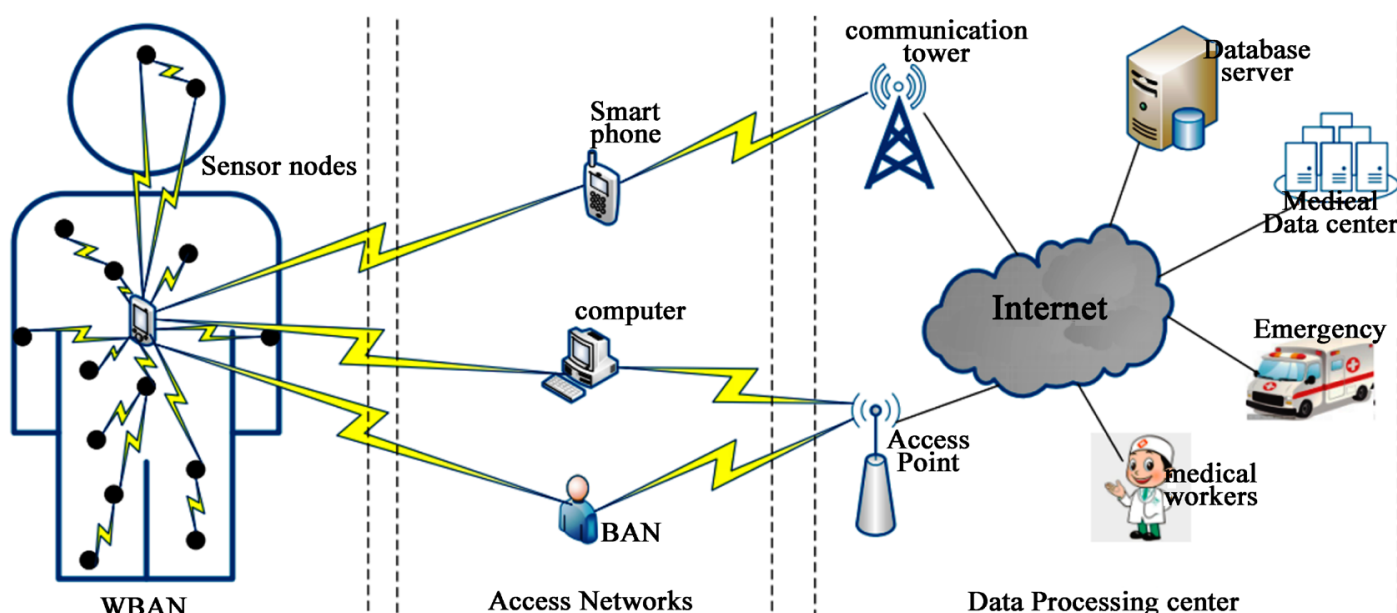

Figure 1. Wireless body area network. 
send frames comparatively more frequently that sometimes resulting in exaggerated channel estimation error. The IEEE 802.15.6 unit has the difficult readying problems with WBANs and has developed a new customary for targeting wireless communications in and around the soma space. It defines because the physical and link layers for WBANs, however, don't specify a TPC rule for WBANs [5].

The device nodes in the WBAN unit are powered by energy-restricted batteries, so reducing energy consumption becomes a necessary issue to be addressed. Although power consumption decreases with the event of low-power physical science technology, the energy efficiency drawback can't be ignored for WBAN with the most transmission distance of $3 \mathrm{~m}$ or $5 \mathrm{~m}$ inside the past few years, a variety of energy-saving ways in which and measures square measure planned and studied. The proposed model associated with energy efficiency rule is expected to minimize the transmission energy of communication systems well as increase the transmission length time [6].

\section{Methodology}

WBAN will works in two ways in the initial stage.

a) Sensor Node;

b) Gateway Node.

1) Sensor Nodes

Sensor nodes are implanted on the human body. They are placed on the surface or inside the body and gather information from the human body and pass it through gateway nodes. Figure 2 shows a dummy illustration of sensor nodes implanted on the surface of the human body.

\section{2) Gateways Node}

The gateway node shown in Figure 3 is responsible for conveyance device info to medical servers or doctors. Failure of the entry node suggests that failure of the WBAN system of a patient. Thus, extending the lifetime of the gateway node may be a fascinating objective in coming up with WBAN systems [7].

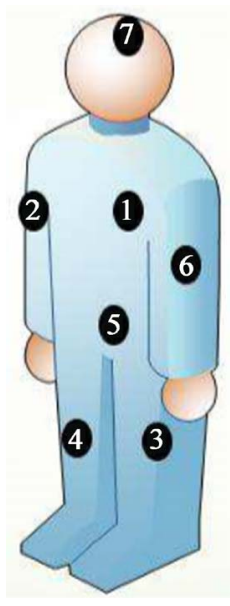

Figure 2. Sensor node in the human body. 
WBAN is designed specifically for medical systems and for emergency things. In such things, the little detector nodes within the network collect very important data using a sensor from the body and upload it to the medical server within the hospital. Figure 4 shows a typical WBAN architecture. This data is then examined by a doctor or a medico within the hospital for identification. The communication within the WBAN is secured as a result of this network uses physiological values (PVs) that are personal human information. This may alter higher attention facilities. Lack of security measures can result in incorrect detection that is risky for human life.

WBAN uses cryptographical keys to secure communication. Bluetooth key distribution theme is employed that is of various sorts.

The main difference between WBAN and WSN has a power and memory management system. The security protocol also is different among them. Security issues also major in WBAN due to monitoring accurately. The most drawback with this security theme is that the risk (of data of knowledge) leak as throughout key agreement this theme exchange quite needed information.

For example, the whole feature sets square measure changed between human action sensors mistreatment this theme. If the sender and also the receiver have

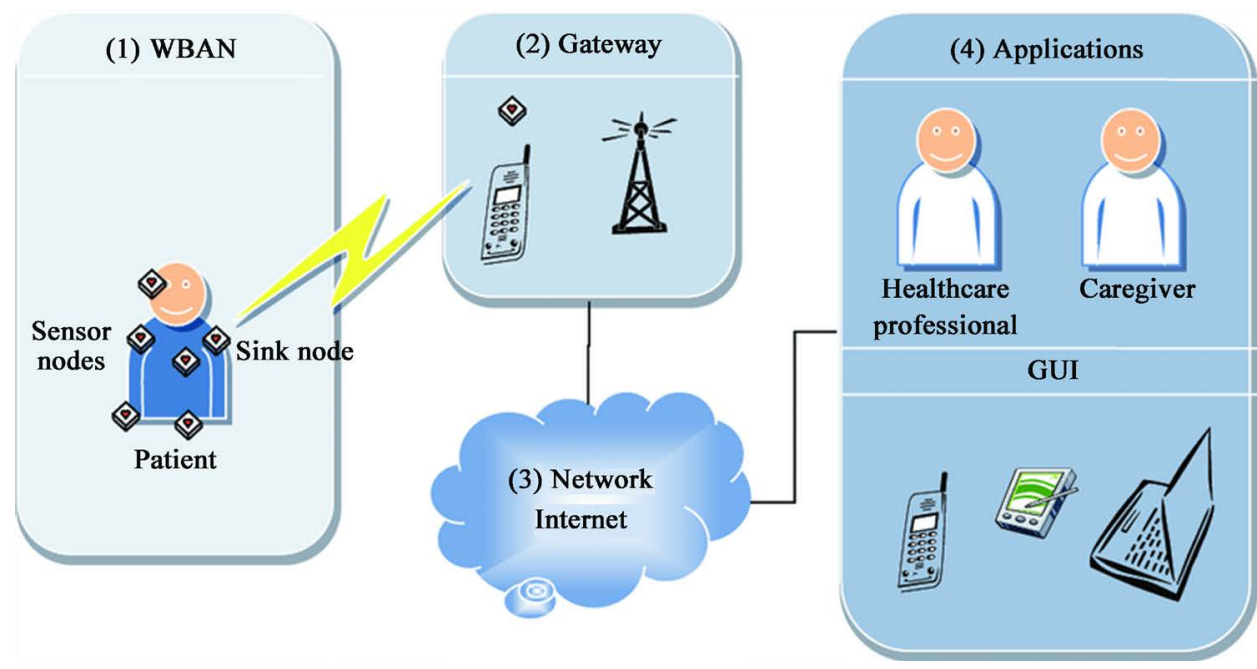

Figure 3. Gateway node.

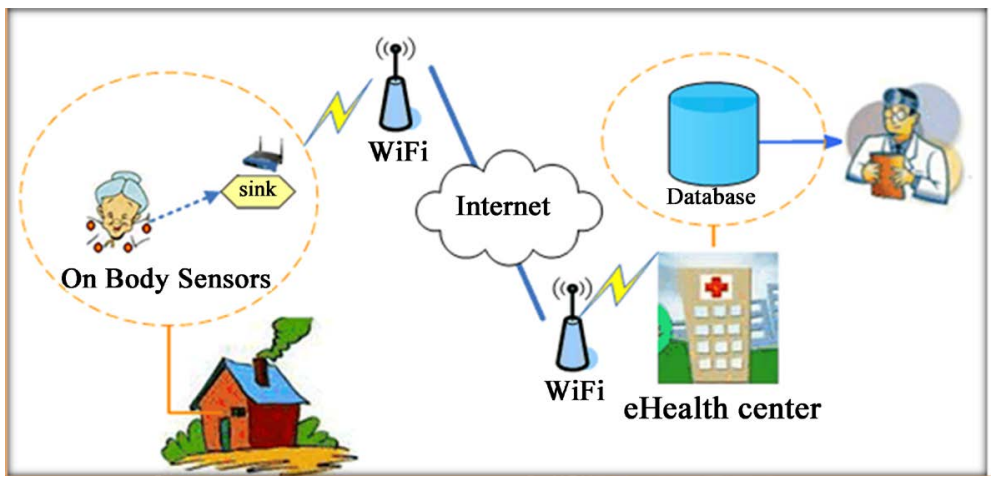

Figure 4. Architecture of WBAN. 
the feature sets the same, then they'll use a standard key. This key may be simply generated by AN outsider World Health Organization acquires of these feature sets [8].

\section{Transmission Power Control}

The TPC section in WBAN is a major part of a model due to the power consumption issue. To design WBAN model power efficiency is given the most priority. For that reason, TPC management needed to improve. It can be done by three steps [9]:

- At first, we need to improve the constancy of a link. A constant link increases transmission power.

- Secondly, the nodes that are connected in a constant area need to minimize collision among the interconnected nodes. More collisions reduce transmission power.

- Finally, proposed transmission power is given to the model for better performance of the channel. Here a good modulation technique works best for energy saving. Controlled transmission power is preferable here.

1) The ZigBee Stack.

A simple view of ZigBee showed in Figure 5. This system will be worked out in our simulation.

- Physical Layer (PHY);

- Madia Access Control (MAC);

- Network (NWK);

- Application Support (APS);

- Security Service Provider (SSP);

- ZigBee Device Object (ZDO);

- Application Framework (APF).

2) Bio Signal Packet Transmission:

A bio-signal is collected from the human body using a sensor node. Medical adjusted with a specific area in the human body. According to the condition of
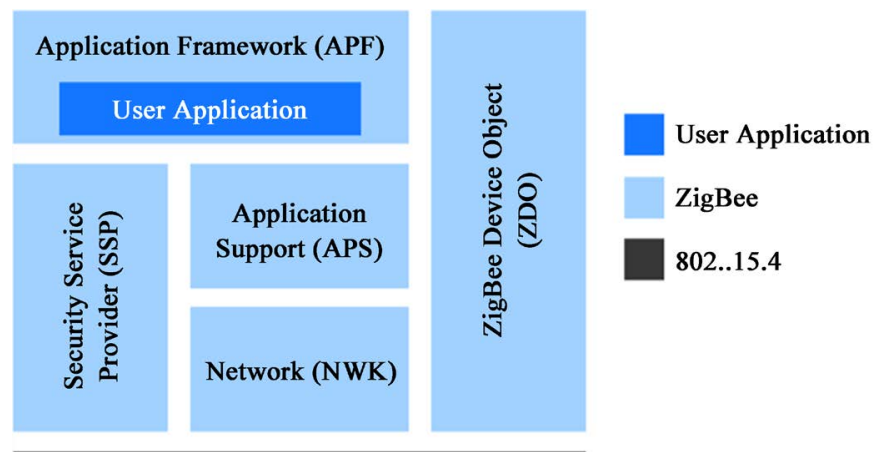

Media Access Control (MAC)

Physical (PHY)

Figure 5. The Zigbee Stack. 
the patient these signals are divided into three categories [10].

a) Urgent: Data collected from the critical condition of a patient is called urgent data. For example, a patient's blood pressure is $160 \mathrm{mmHg}$ or getter, this data from the blood pressure sensor is called urgent data. It is given the most priority because this condition is more sensitive. For this reason, this condition sensor node collects data continuously.

b) Semi-urgent: This type of bio-signal some less important than an urgent data signal. For example, ECG gives the result of heartbeats near $60 \mathrm{bpm}$. It is not normal but more important the normal. At any time have a chance to go a patient critical condition. This condition is given medium priority.

c) Non-urgent: This type of bio-signal is considered less important. When a patient is in a normal condition or a completely healthy man, it is not important to measure his blood pressure continuously. The sensor node can be given a time interval to reduce power consumption.

\section{Implementation and Results}

Simulations and results demonstrate the performance of star, mesh, cluster-tree topologies in different performance parameters and those are throughput, delay, load, data traffic sent, data traffic received, packet dropped so that simulation and result part is an important approach to evaluating the work. To develop a real-time scenario of a project work instead of simulation model work it must be requiring more time, financial cost, and effort through a simulation model that doesn't need to present necessarily appropriate. But the main object of any simulation model is to properly portend the behavior of real scenarios [11].

For design ZigBee model many simulators can be proposed but, in this research, used OPNET simulator and OMNET++ for analysis power control. OPNET has an academic version known as Riverbed and OMNET++ is an open-source network simulator that represents graphically interface.

In this paper, the OPNET is used to design mesh, star, and cluster topologies with $250 \mathrm{kbps}$ and $1024 \mathrm{kbps}$ data rate. In this project work was designed with ZigBee sensor nodes.

1) Scenario: Simulation of different topologies at $250 \mathrm{kbps}$ data rate in the noiseless area

Firstly, here is discussed with 6 sensor nodes and $250 \mathrm{Kbps}$ data rates for mesh topology. Here is considered a hospital environment like ICU. Here noise is too low and it fixed around $40 \mathrm{kbps}$ per sensor. For 6 sensors need around $250 \mathrm{kbps}$ calculated. Finally, the amount of data transmitted will be $2025 \mathrm{kbps}$ per node.

Figure 6 contains star topology with 6 sensor nodes, router, ZigBee coordinator, and user applications. Star topology applied with a $250 \mathrm{kbps}$ data rate.

Figure 7 contains 6 sensor node, router, ZigBee coordinator. Cluster topology is applied here with a $250-\mathrm{kbps}$ data rate.

a) Throughput

Throughput describes how fast the total number of data successfully sends 


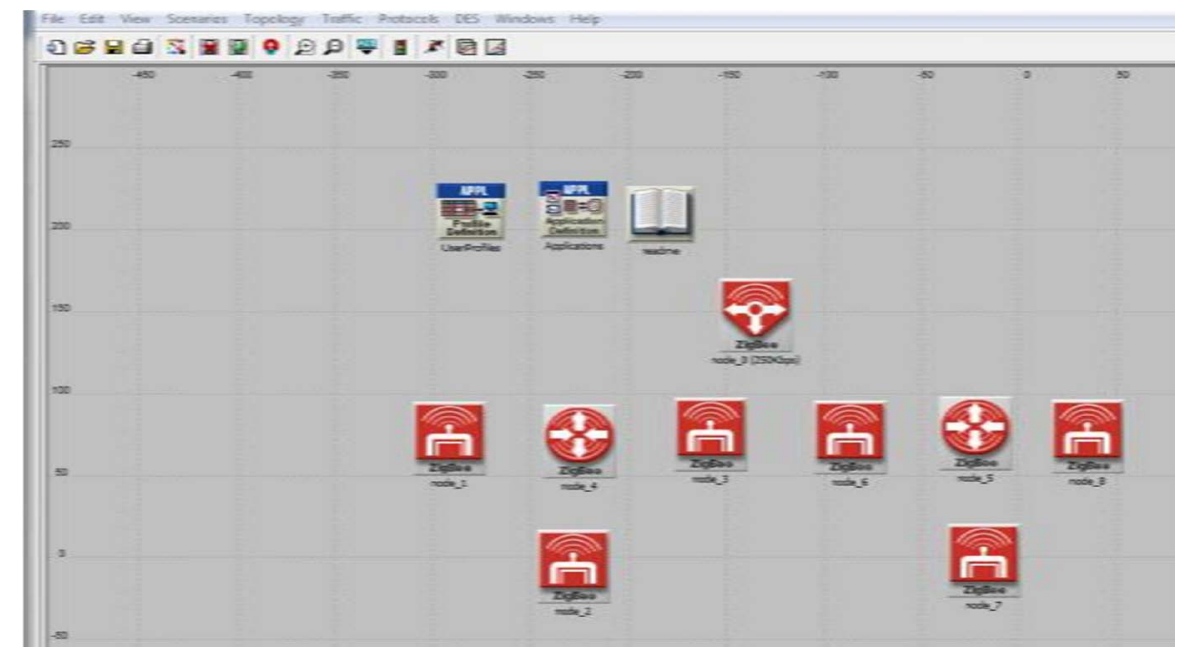

Figure 6. Star topology with 250kbps data rate.

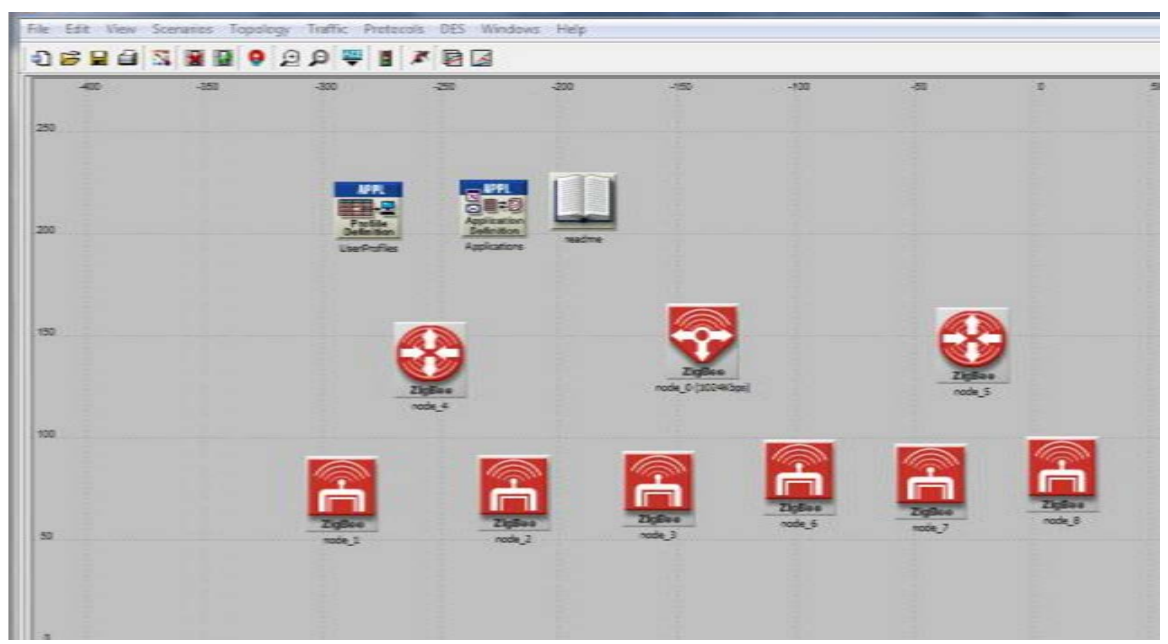

Figure 7. Cluster-tree topology with 250kbps data rate.

and received a physical layer from MAC. Throughput also is the actual measurement of data send using communication channels [12].

Figure 8 represents that which topology gives better performance. In 1224 seconds, the maximum throughput values have been obtained $59.22 \mathrm{kbps}, 94.16$ kbps in 1764 second, and $63.49 \mathrm{kbps}$ in 918 seconds for the cluster, mesh and star topologies respectively.

b) Delay

The delay means the amount of extra time compares with actual processing time on the communication channel. It reduces channel accuracy or efficiency. A small amount of delay prefers for better performance [13].

In above Figure 9, the minimum delay has been recorded in the cluster, mesh, and star topologies are $0.0128 \mathrm{sec}, 0.0127 \mathrm{sec}$, and $0.0143 \mathrm{sec}$ respectively. The minimum delay of these topologies gives the best result.

c) Load

Load refers to the amount of total data traffic being carried among the 


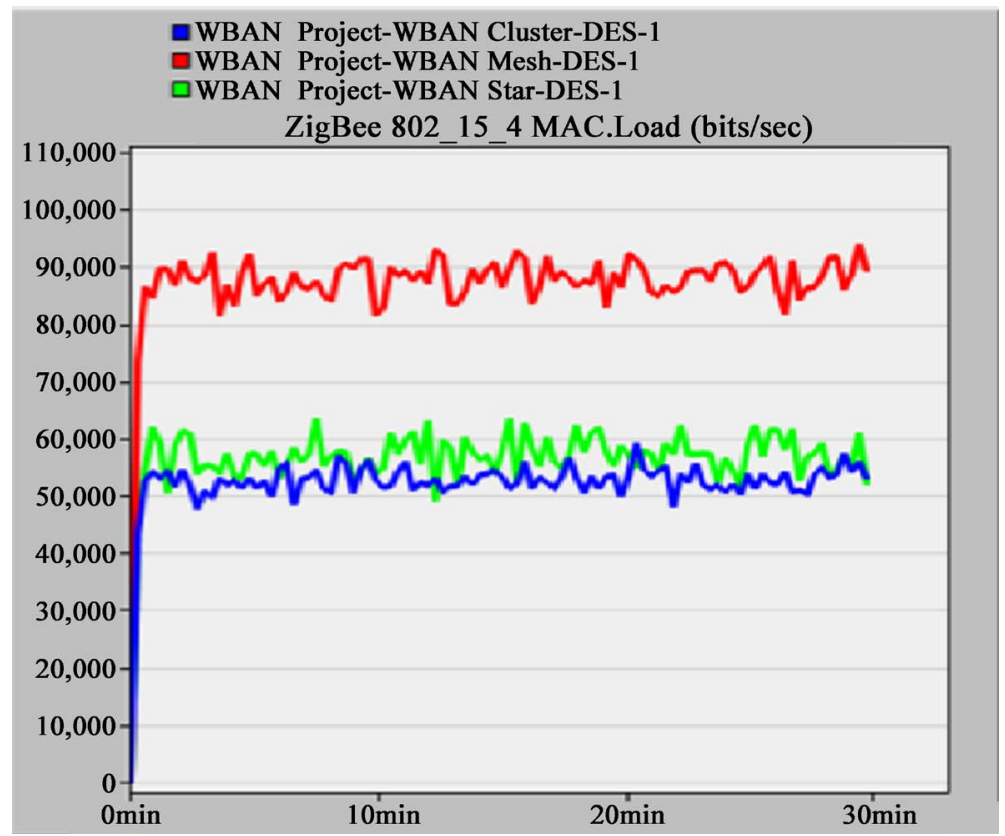

Figure 8. Throughput for $250 \mathrm{kbps}$ data rate.

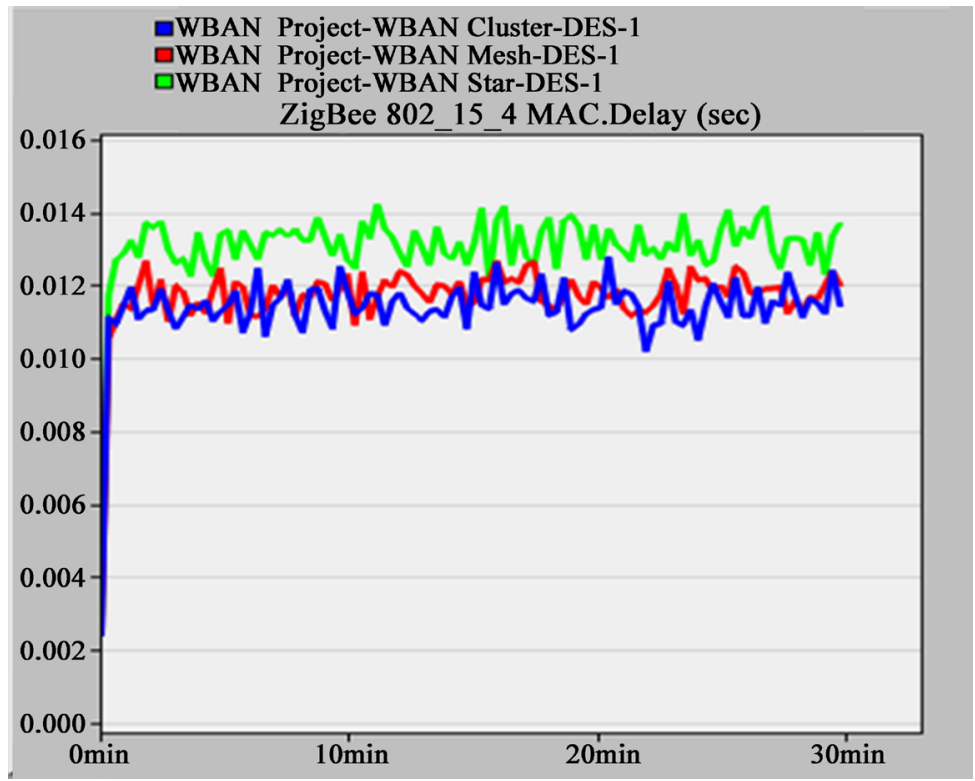

Figure 9. Delay for different topologies in the $250 \mathrm{kbps}$ data rate.

communication channel in data communication. The performance of a communication channel will be better at a low load.

In Figure 10, it has been shown the amount of load with $250 \mathrm{kbps}$ data rate in the cluster, mesh, and star topologies. The maximum load is shown in mesh topology $29.175 \mathrm{kbps}$ and cluster, star topology respectively $22.925,22.865 \mathrm{kbps}$.

d) Data Dropped for Different Data Rate

The amount of data that cannot be transmitted through a channel called data dropped. It was held due to failure ACK of the MAC protocol for retransmit data. A large number of data dropped to reduce the performance of a channel. 
In Figure 11 when time starts 0-sec data dropped was Zero and at $1782 \mathrm{sec}$ data dropped was $1.519 \mathrm{kbps}$ for the cluster, $3.544 \mathrm{kbps}$ for mesh, $4.923 \mathrm{kbps}$ for the star.

e) Data Traffic Sent

Data traffic is that the quantity information of knowledge of information moving across a network channel. The amount of data traffic sent measured the performance of a channel. As much as the high amount of data traffic sent this

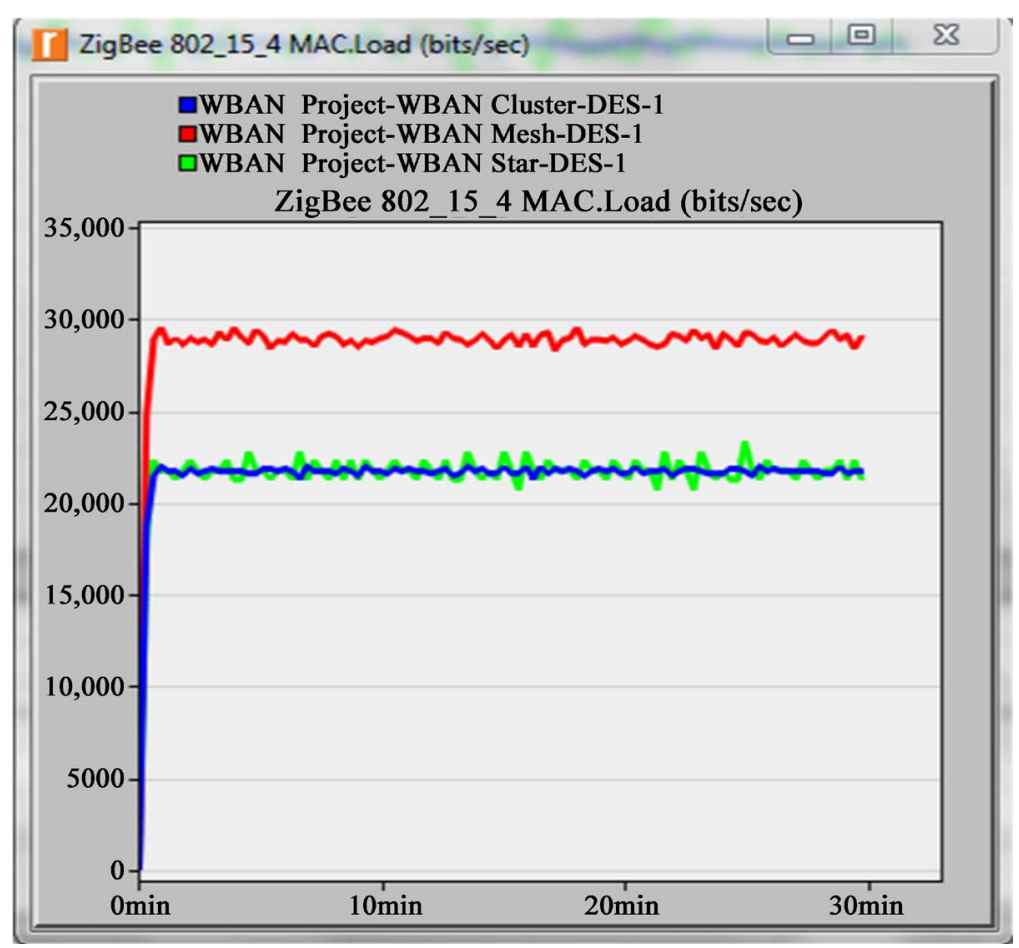

Figure 10. Load for different topologies in the $250 \mathrm{~kb}$ data rate.

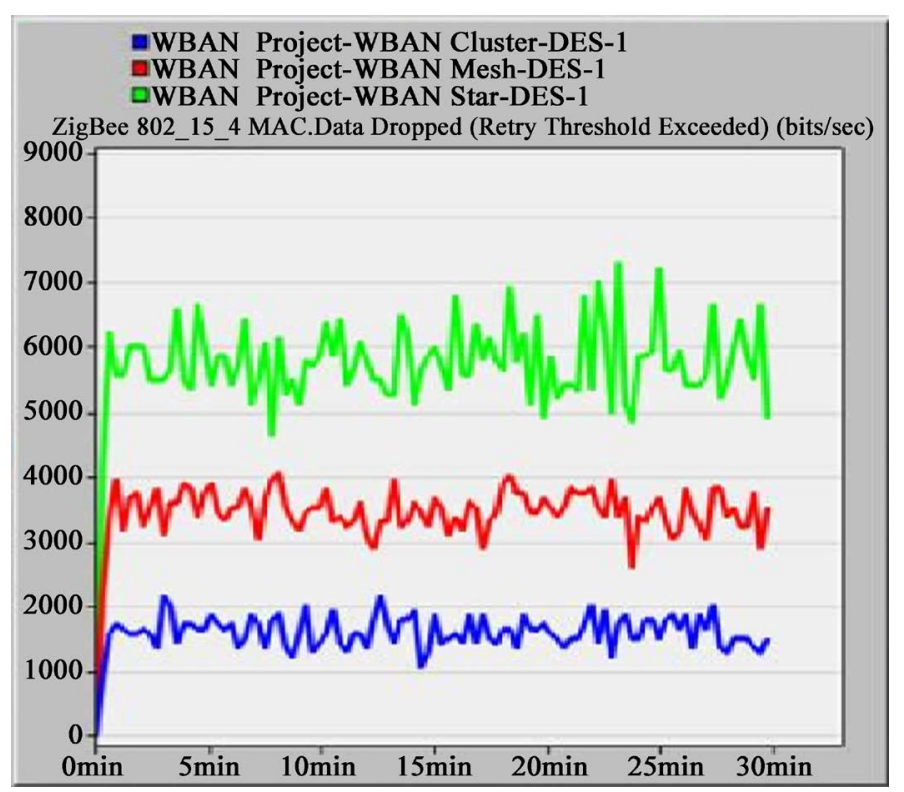

Figure 11. Data dropped for different data rate. 
result will be best.

For $250 \mathrm{kbps}$ data rate the Figure 12 is has been shown that cluster-tree gets $23.32 \mathrm{kbps}, 29.320 \mathrm{kbps}$ for mesh and star gets $18.51 \mathrm{kbps}$ data traffic received by MAC from the physical layer.

2) Scenario 2: Simulation of different topology at $1024 \mathrm{kbps} / \mathrm{Mbps}$ data rate

Figure 13 contains 6 sensor node, ZigBee coordinator, user applications, etc. Here used a 1024 kbps data rate with a mesh topology.

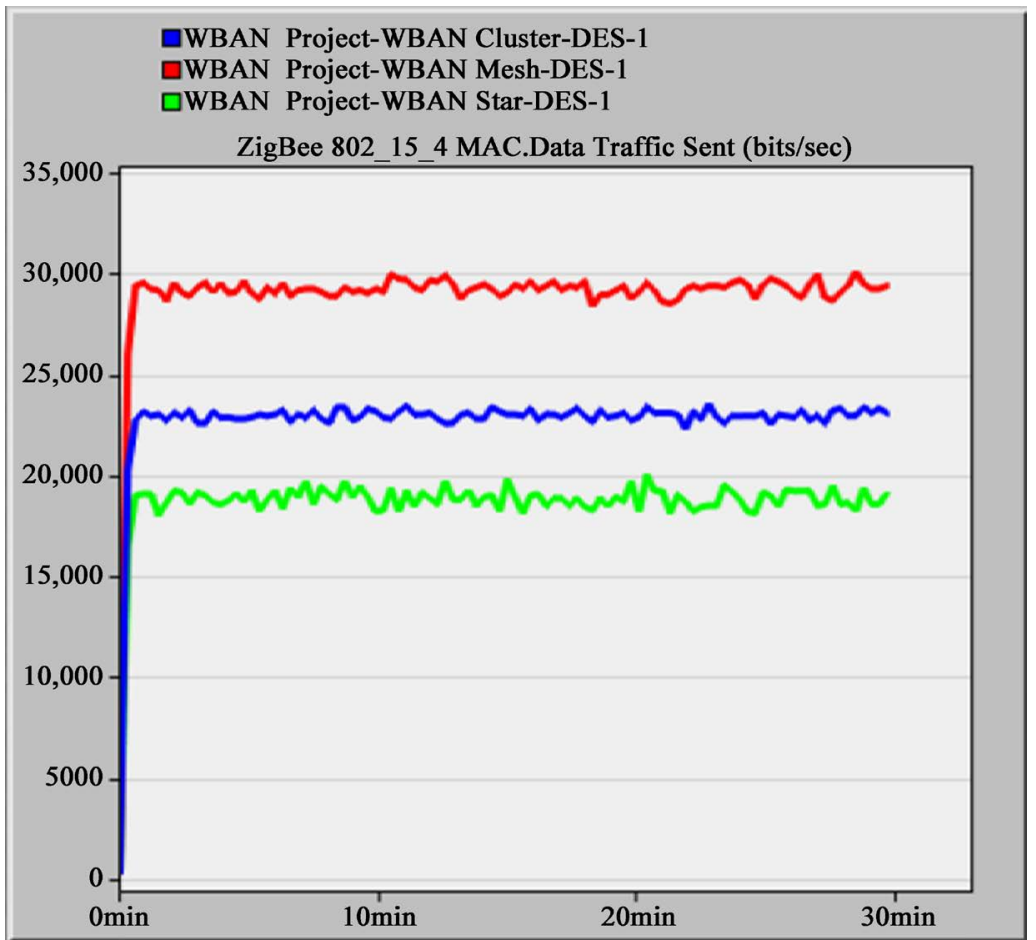

Figure 12. Data traffic received.

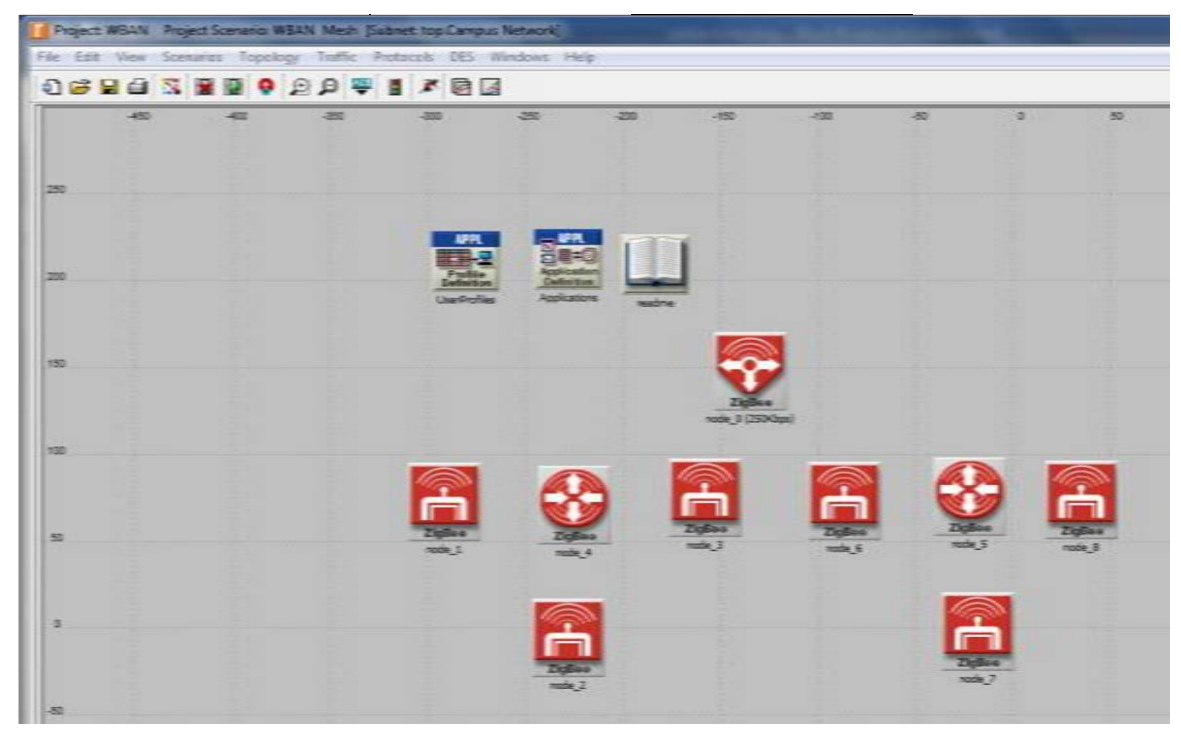

Figure 13. Mesh topology with $1024 \mathrm{kbps}$ data rate. 
Figure 14 contains 6 sensor nodes, ZigBee coordinator, user applications, etc. Here used a $1024 \mathrm{kbps}$ data rate with star topology.

Figure 15 contains 6 sensor node, ZigBee coordinator and user applications, etc. Here used a $1024 \mathrm{kbps}$ data rate with cluster topology.

\section{a) Throughput}

In Figure 16, the maximum throughput values have been obtained in 64.088 kbps in 90 seconds for the cluster, $55.372 \mathrm{kbps}$ in 36 seconds for mesh, and 50.17 kbps in 72 seconds for star topologies.

\section{b) Delay}

In Figure 17, the minimum delay has been recorded in the cluster, mesh, and star topologies are $0.012111 \mathrm{sec}, 0.009399 \mathrm{sec}$, and $0.01360035 \mathrm{sec}$ in 18,18 , and $36 \mathrm{sec}$ respectively.

\section{c) Load}

In Figure 18, the maximum load has been recorded in the cluster, mesh, and star topologies are $29.200 \mathrm{kbits} / \mathrm{sec}, 23.555 \mathrm{kbits} / \mathrm{sec}$, and $12.672 \mathrm{kbits} / \mathrm{sec}$

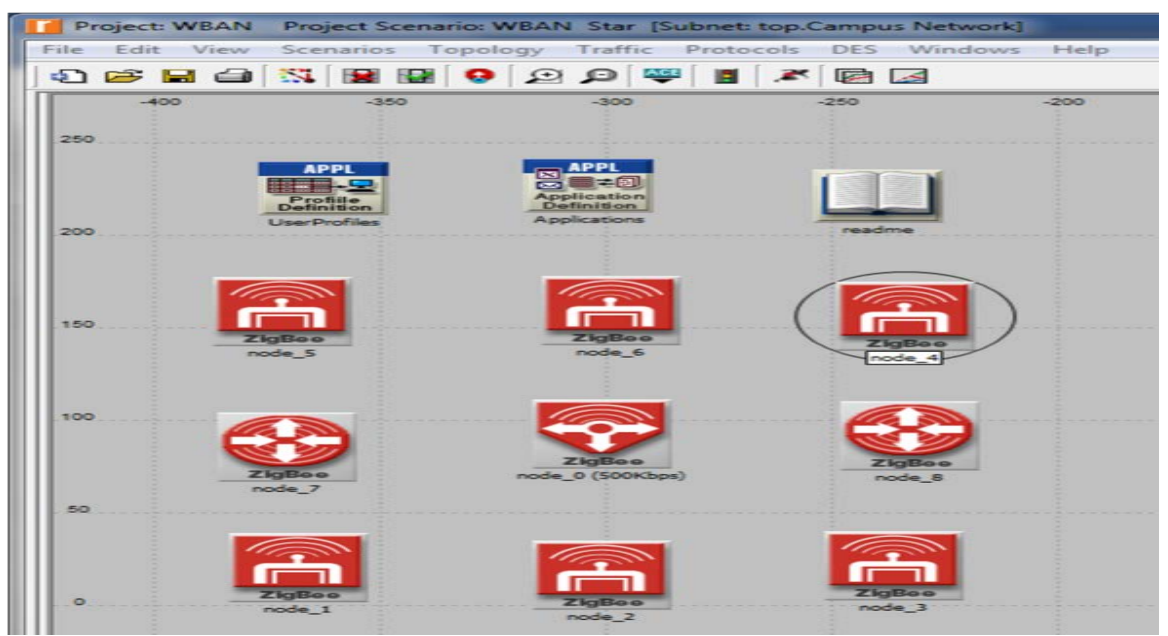

Figure 14. Star topology with $1024 \mathrm{kbps}$ data rate.

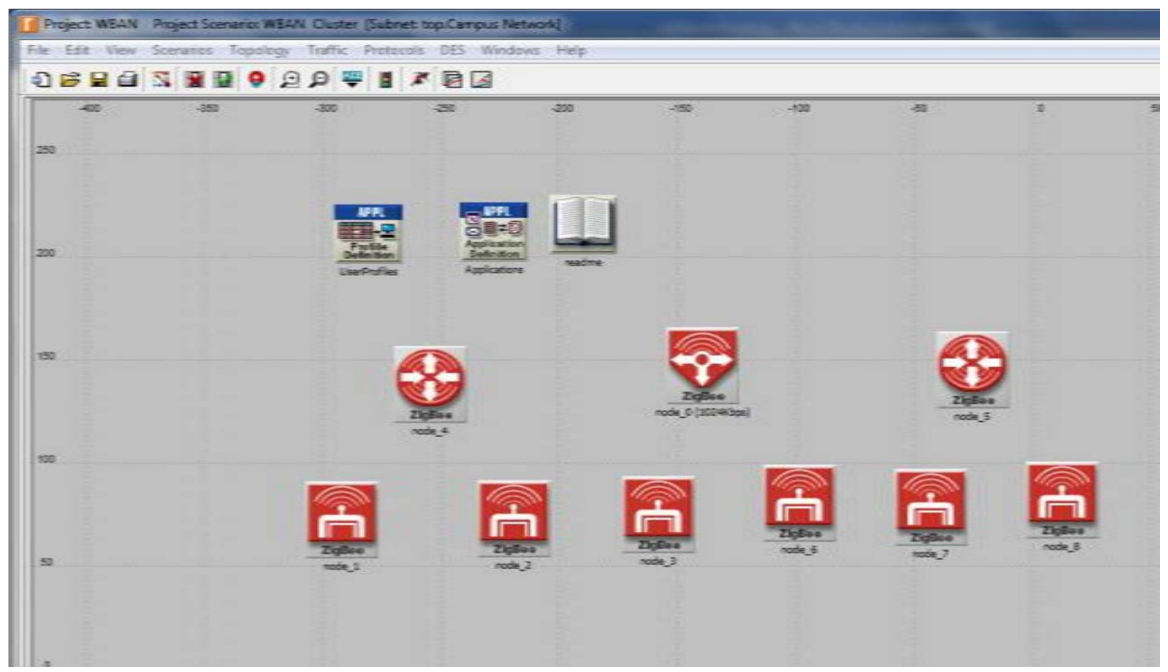

Figure 15. Cluster topology with $1024 \mathrm{kbps} / 1 \mathrm{Mbps}$ data rate. 


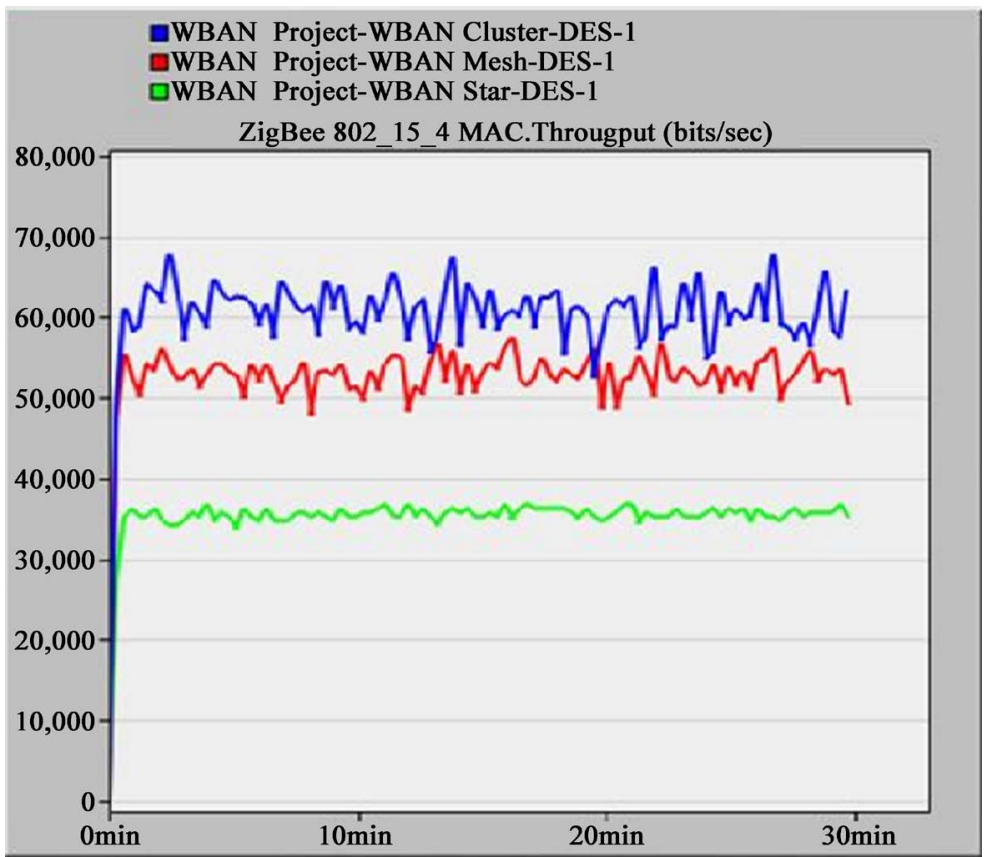

Figure 16. Throughput for different topologies in $1024 \mathrm{kbps}$ Data rate.

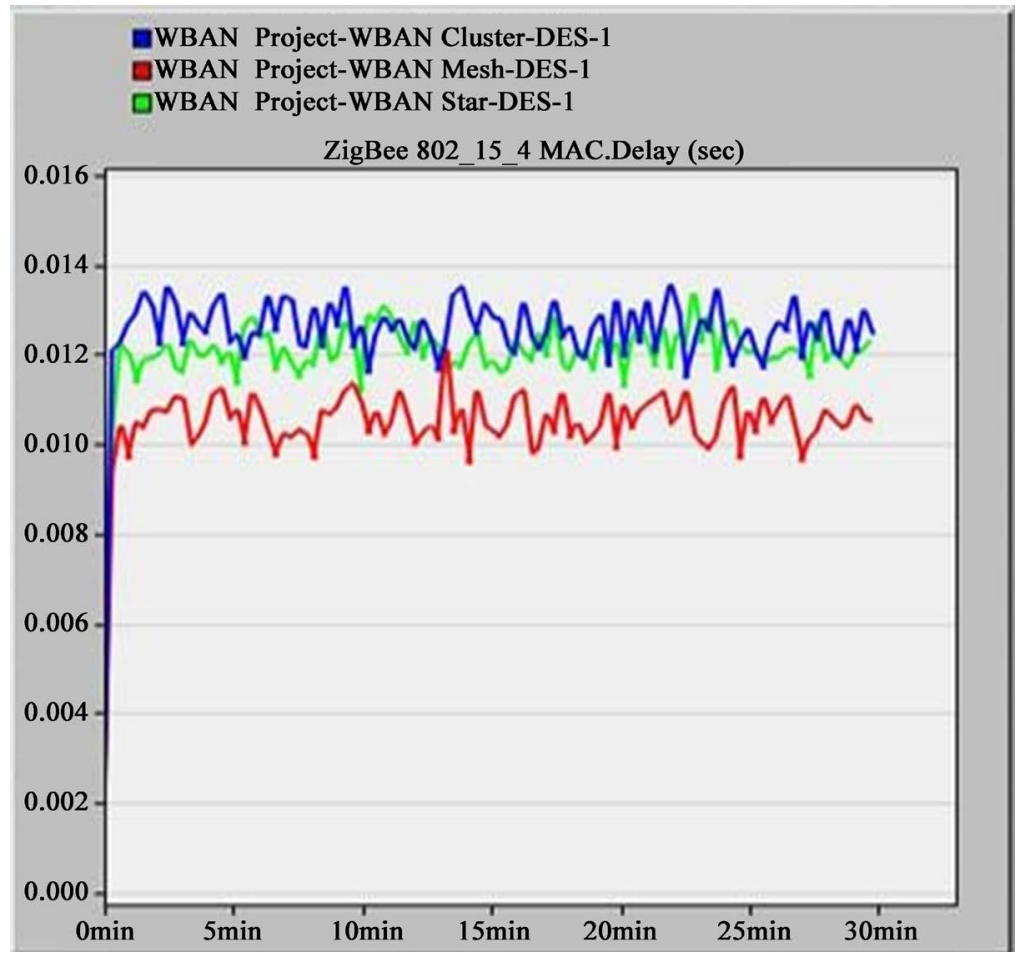

Figure 17. Delay for different topologies in $1024 \mathrm{kbps}$ Data rate.

in 108,72 and $126 \mathrm{sec}$ respectively.

\section{d) Packet drop Dropped}

In this Figure 19, the value of packet drop has been gained in the cluster, mesh, and star topologies are $513.87 \mathrm{bits} / \mathrm{sec}, 956.4444 \mathrm{bits} / \mathrm{sec}$, and 4274.2222 bits/sec in $18 \mathrm{sec}$ respectively. 


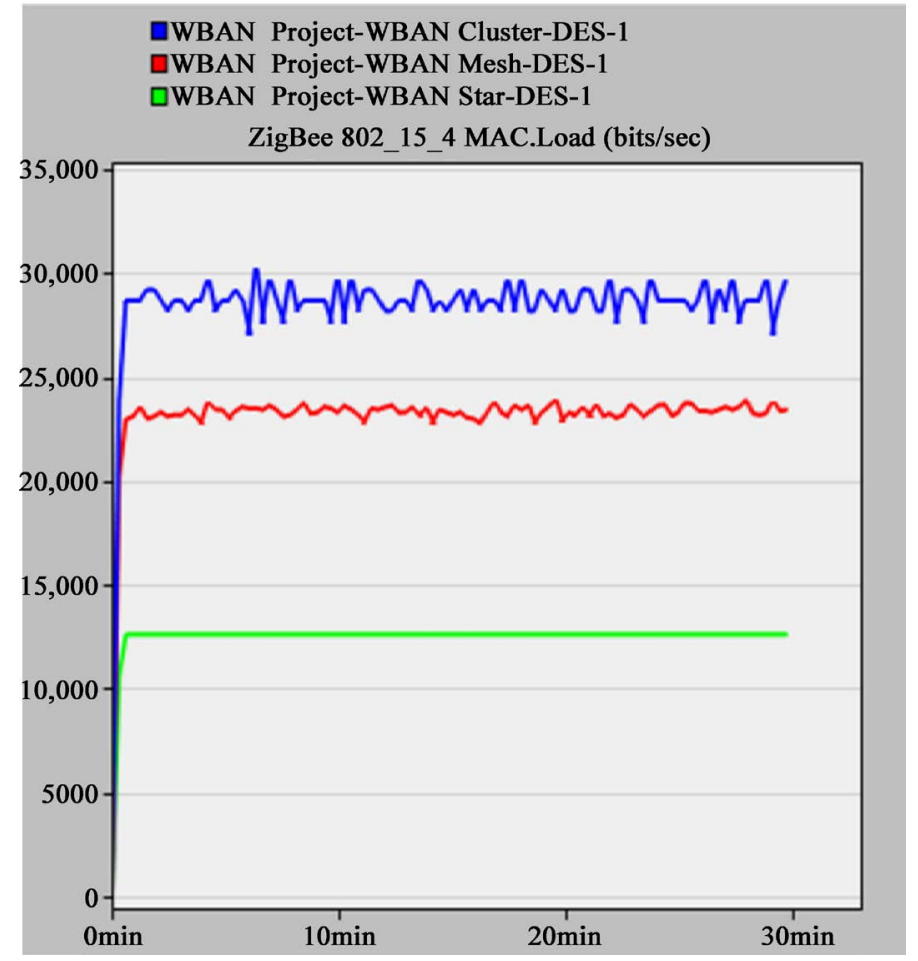

Figure 18. Load for different topologies in $1024 \mathrm{kbps}$.

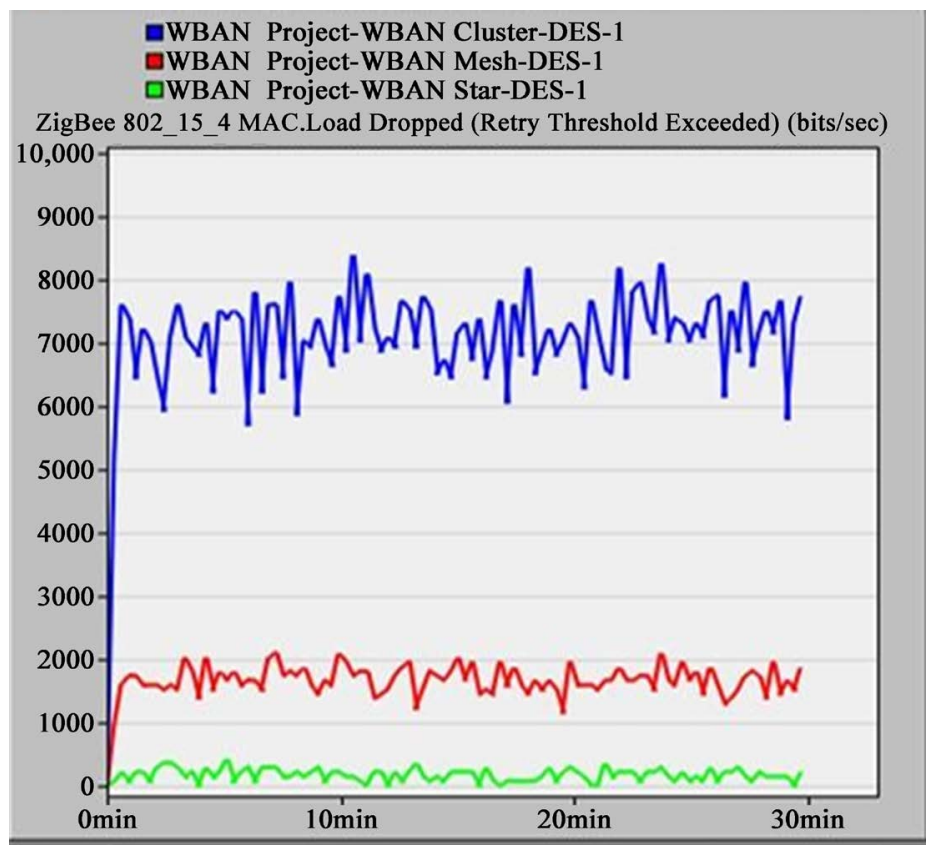

Figure 19. Packet drop for different topologies in $1024 \mathrm{kbps}$ Data rate.

\section{e) The Packet Sent}

In above Figure 20, packet sent for cluster-tree $26.40 \mathrm{kbps}$, mesh $25.95 \mathrm{kbps}$ and star $14.3 \mathrm{kbps}$ at $379 \mathrm{sec}, 1171 \mathrm{sec}$, and $1170 \mathrm{sec}$ respectively.

\section{f) Packet Received}

In above Figure 21, cluster-tree 197.359 kbps, mesh 204.17 kbps and star 


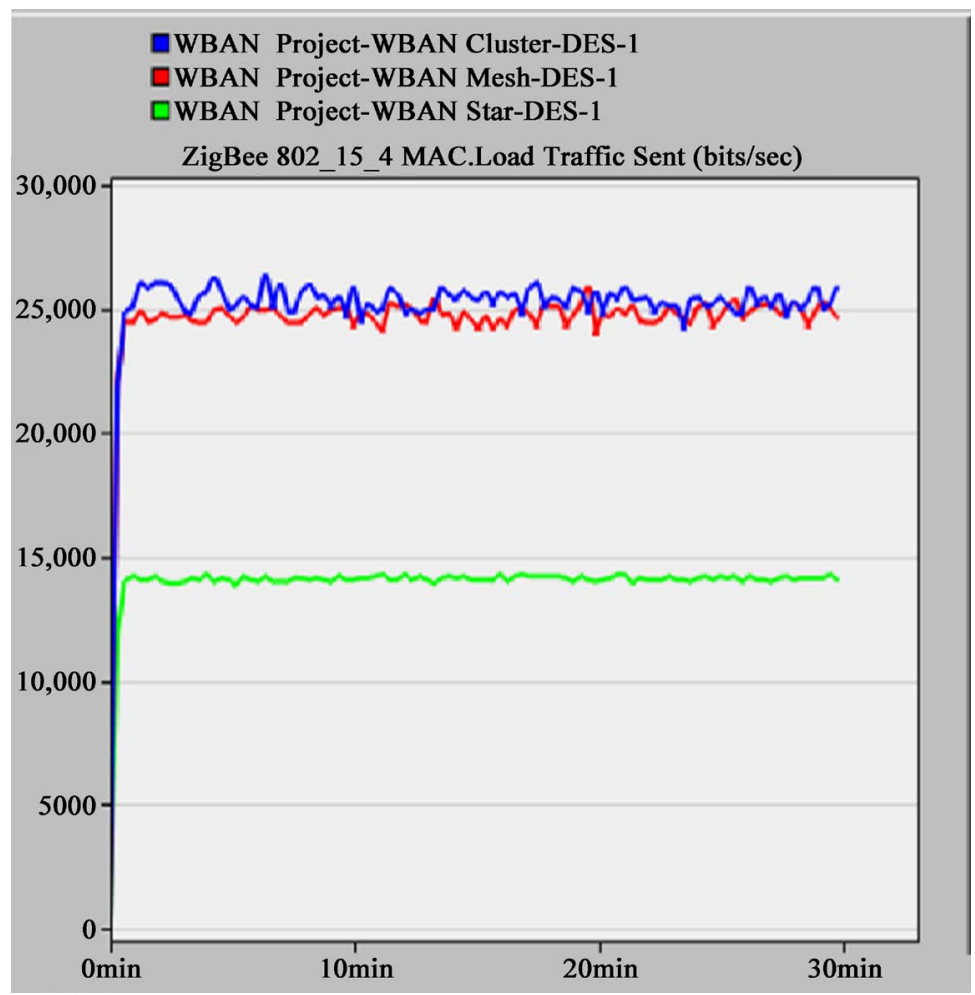

Figure 20. Data sent for different topologies in $1024 \mathrm{kbps}$ Data rate.

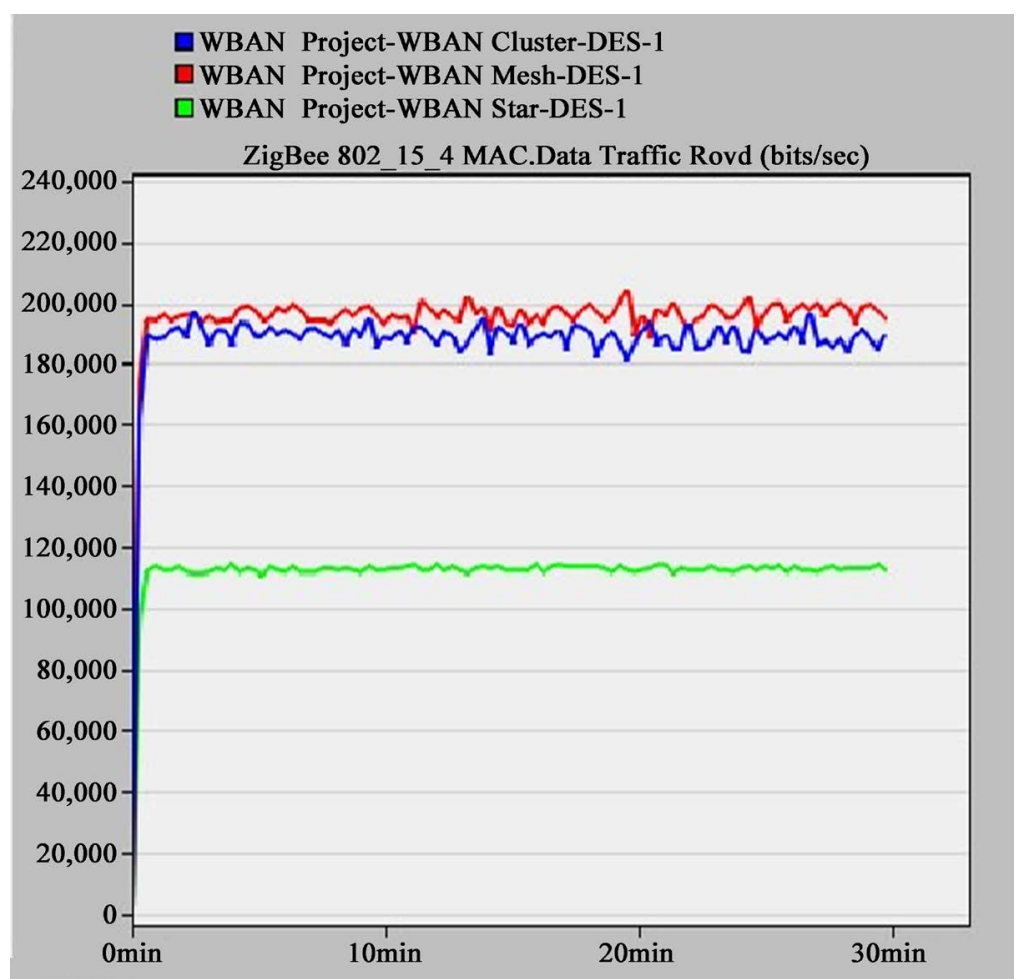

Figure 21. Packet receive for different topologies in $1024 \mathrm{kbps}$.

$118.11 \mathrm{kbps}$ received data at $144 \mathrm{sec}, 1171 \mathrm{sec}$, and 666 secs respectively.

3) Scenario 3: Simulation with data segmentation techniques 
There are several techniques used in WBAN technology. Data segmentation is one of them. In this technique data, it is classified into three categories according to the importance of data types. This scenario compared the data segmentation technique compared with other two existing techniques [3] [6]. Table 1 gives the different parameters and values to configure all parameters.

\section{PACKET DELIVERY RATIO}

The packet delivery ratio means the number of packets is different from the transmitter and receiver. It ensures channel quality.

Figure 22 shows that packet transmission in simulation time $1000 \mathrm{~s}$, the 2000 s, and $3000 \mathrm{~s}$. The power consumption rate increasing is with simulation time rapidly. The data classification method is better than the existing QoS-aware and Anomaly detection method.

Figure 23 shows that power consumption of oxygen sensor increasing according to simulation time. Data segmentation techniques reduce more data transmission increasing simulation time as like sleeping method.

In Figure 24, the power consumption of the blood pressure sensor has been shown. Here the power consumption has a significant reduction in the proposed system comparing two reference models. Performance Analysis is the method of learning or evaluating the performance of a selected situation as compared

Table 1. The configuration of parameters.

\begin{tabular}{cc}
\hline Parameter & Value \\
\hline Arrangement type & Static (transmission. continuously) \\
Sensor loads & loads $1.0 \mathrm{p} / \mathrm{s}, .2 \mathrm{p} / \mathrm{s}$, and $10 \mathrm{p} / \mathrm{s}$ \\
No. of sensor & 1 \\
Sensor Energy & $100 \mathrm{~mW}$ \\
Gateway Energy & $100 \mathrm{~mW}$ \\
Buffer packet size & One packet \\
Simulation time & $1000 \mathrm{~s}, 2000 \mathrm{~s}, 3000 \mathrm{~s}$ \\
Sensor Type & ECG, SpO2 and Blood pressure
\end{tabular}

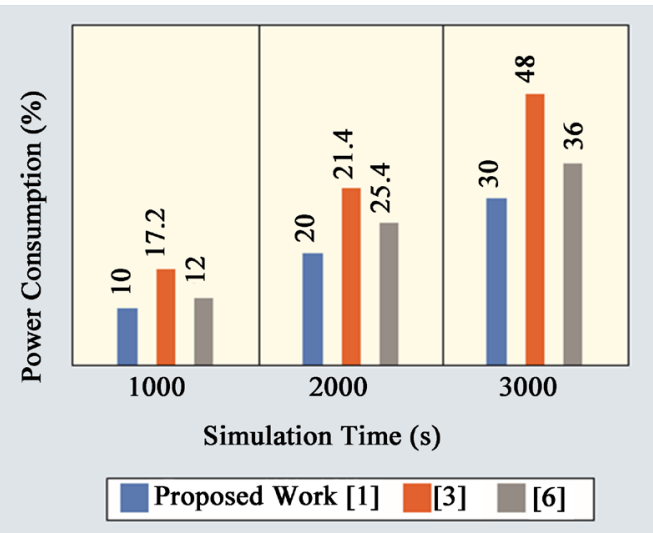

Figure 22. Packet delivery ratio. 
to the target that was to be achieved. Performance analysis is often neutralizing finance on the premise of ROI, profits, etc. Here performance will be analyzed for differences in real-time scenarios. In the first scenario mesh, star, and cluster topologies are simulated in the $250 \mathrm{kbps}$ data rate.

Figure 25 shows that, the performance of mesh topology is best for data transmission. The efficiency of a channel reduces transmission power consumption. So, mesh topology is the best to consider TPC analysis.

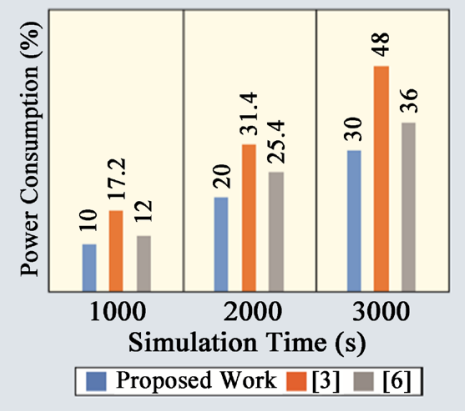

Figure 23. Power consumption in the oxygen sensor.

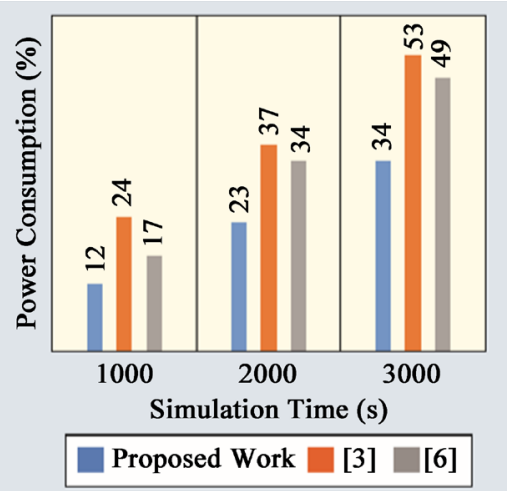

Figure 24. The power consumption in the blood pressure measuring sensor.

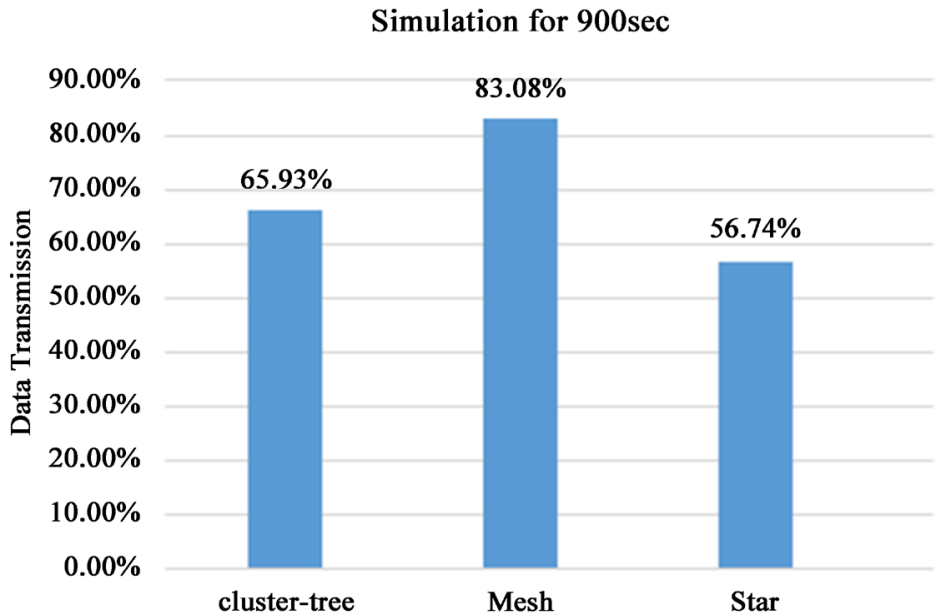

Figure 25. Data transmission through scenario 1. 


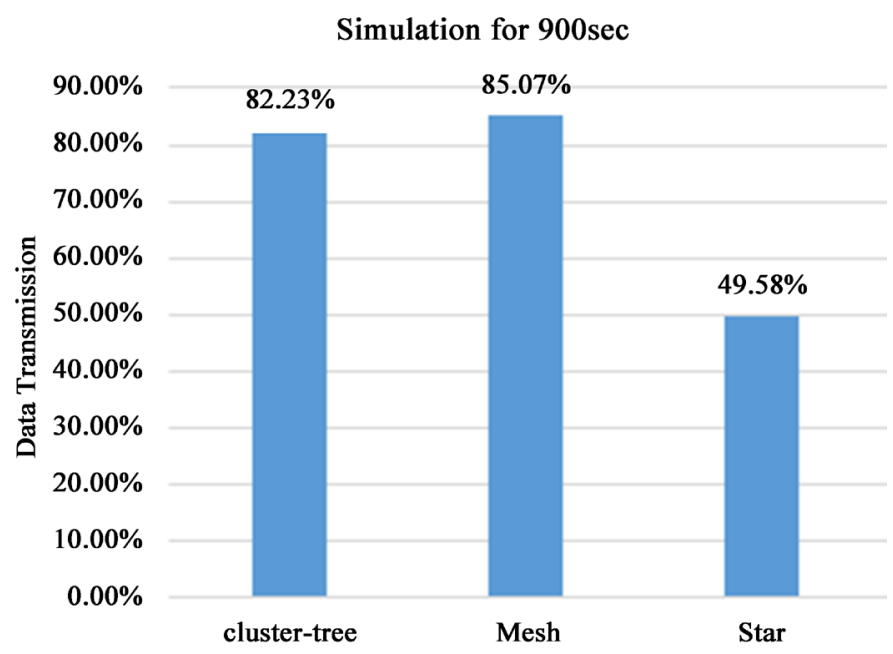

Figure 26. Data transmission through scenario 2.

In data of Figure 26, the rate was the same $(1 \mathrm{mbps})$ for cluster-tree, mesh, and star topology but data transmission rate better in Mesh.

\section{Conclusion}

A new simulation methodology of WBANs transmission power control analysis has been presented in the paper. In the first scenario mesh, star, and cluster topologies are simulated in $250 \mathrm{kbps}$ data rate using ZigBee at different topologies. Only three topologies are used here because the IEEE802.15.4 standard (ZigBee) only supports the cluster, mesh, star topologies. Here focused on the power control analysis of ZigBee sensor nodes using OPNET and OMNET++ simulators in this paper. To test the performance of the topologies, this paper simulated or analyzed them for different types of scenarios. Here analysis with different data rates for creating a different environmental situation and find low power consuming topology. Mesh topology is best to consider TPC analysis for throughput, data traffic sent and received. Also, for $1024 \mathrm{kbps}$ data rate mesh was better for the delay and data traffic received. So it can be said that mesh topology is the best. The data segregation technique is also more effective for reducing power consumption. The results outperform the best model to reduce power consumption that extends battery life. In wireless technology another major subject is security. Violate attacks may be a dangerous issue for WBAN medical services or others. The amount of power consumed can be increased due to gateway failure. By implementation of a collision reduce the technique between the warning and welcome back packets of the gateway to improving the packet delivery ratio of the introduced gateway failure algorithm. Also, develop a Security protocol is a challenge for WBAN.

\section{Conflicts of Interest}

The authors declare no conflicts of interest regarding the publication of this paper. 


\section{References}

[1] Zhang, H. and Safaei, F. (2018) Channel Autocorrelation-Based Dynamic Slot Scheduling for Body Area Networks. EURASIP Journal on Wireless Communications and Networking, 2018, Article No. 246. https://doi.org/10.1186/s13638-018-1261-8

[2] Archasantisuk, S., Aoyagi, T., Kim, M. and Takada, J.I. (2016, September) Transmission Power Control in WBAN Using the Context-Specific Temporal Correlation Model. 2016 IEEE 27 th Annual International Symposium on Personal, Indoor, and Mobile Radio Communications (PIMRC), Valencia, Spain, 4-8 September 2016, 1-6.

[3] Archasantisuk, S., Aoyagi, T., Kim, M. and Takada, J.I. (2018) Temporal Correlation Model-Based Transmission Power Control in a Wireless Body Area Network. IET Wireless Sensor Systems, 8, 191-199. https://doi.org/10.1049/iet-wss.2016.0109

[4] Fernandes, D., Ferreira, A.G., Abrishambaf, R., Mendes, J. and Cabral, J. (2017) Survey and Taxonomy of Transmissions Power Control Mechanisms for Wireless Body Area Networks. IEEE Communications Surveys \& Tutorials, 20, 1292-1328. https://doi.org/10.1109/COMST.2017.2782666

[5] Di Franco, F., Tachtatzis, C., Atkinson, R.C., Tinnirello, I. and Glover, I.A. (2014) Channel Estimation and Transmit Power Control in Wireless Body Area Networks. IET Wireless Sensor Systems, 5, 11-19. https://doi.org/10.1049/iet-wss.2013.0070

[6] Zhengl, Z., Zheng, X., Tian, J. and Shu, M. (2018, May) A Transmission Power Control Algorithm for Wireless Body Area Networks. 2018 IEEE 22nd International Conference on Computer Supported Cooperative Work in Design (CSCWD), Nanjing, China, 9-11 May 2018, 854-858.

[7] Rahman, A. and Khan, N. (2016, August) Optimum Placement of Gateway Node on the Human Body for Real-Time Healthcare Monitoring Using WBAN. 2016 Sixth International Conference on Innovative Computing Technology (INTECH), Dublin, Ireland, 24-26 August 2016, 408-412.

[8] Barakah, D. M. and Ammad-uddin, M. (2012, February) A Survey of Challenges and applications of Wireless Body Area Network (WBAN) and Role of a Virtual Doctor Server in Existing Architecture. 2012 Third International Conference on Intelligent Systems Modelling and Simulation, Kota Kinabalu, Malaysia, 8-10 February 2012, 214-219.

[9] Jafari, R. and Effatparvar, M. (2017) An Overview on the Transmission Power Control. The International Journal of Computer and Information Technologies (IJOCIT), 5, 43-51.

[10] Abiodun, A. S., Anisi, M.H., Ali, I., Akhunzada, A. and Khan, M.K. (2017) Reducing Power Consumption in Wireless Body Area Networks: A Novel Data Segregation and Classification Technique. IEEE Consumer Electronics Magazine, 6, 38-47. https://doi.org/10.1109/MCE.2017.2715518

[11] Denis, B., Amiot, N., Uguen, B., Guizar, A., Goursaud, C., Ouni, A. and Chaudet, C. (2014, December) Qualitative Analysis of RSSI Behavior in Cooperative Wireless Body Area Networks for Mobility Detection and Navigation Applications. 201421 st IEEE International Conference on Electronics, Circuits and Systems (ICECS), Marseille, France, 7-10 December 2014, 834-837.

[12] Benolirao, J.A., de Joya, A.J., Lim, I., Osayta, L.K. and Cordel, M. (2016, May) Quantifying the Throughput and Latency Contribution in Secured IEEE 802.15. 6 WBAN Simulated Transmission. 2016 IEEE Region 10 Symposium (TENSYMP), Bali, Indonesia, 9-11 May 2016, 305-310.

[13] Roblin, C. (2011, September) Analysis of the Channel Power Delay Profile of WBAN Scenarios in Various Indoor Environments. 2011 IEEE International Conference on Ultra-Wideband (ICUWB). Bologna, Italy, 14-16 September 2011, 545-549. 